Schmerztherapie-Vereinbarung

\section{Erleichterter Zugang für Ärzte}

— Der Zugang zur Versorgung chronisch schmerzkranker Patienten wird für Vertragsärzte erleichtert. Das sieht die erneuerte Schmerztherapie-Vereinbarung vor, auf die sich die Kassenärztliche Bundesvereinigung und der GKV-Spitzenverband jetzt geeinigt haben. „Mit der aktualisierten Vereinbarung werden wir mehr Ärzte für die Versorgung chronisch schmerzkranker Patienten gewinnen können", äußerte sich KBV-Vorsitzender Dr. Andre- as Gassen laut Pressemitteilung zuversichtlich. „Die Anpassung an moderne Arbeitszeitmodelle, der Wegfall des obligatorischen Eingangskolloquiums unter bestimmten Voraussetzungen und die Einführung einer befristeten Dokumentationsprüfung sind wichtige Schritte, um den Ärztezugang zu einer qualitätsgesicherten schmerzmedizinischen Versorgung zu erleichtern und damit das Nachwuchsproblem etwas zu entschär- fen", erklärte Professor Joachim Nadstawek, Vorsitzender des Berufsverbands der Ärzte und Psychotherapeuten in der Schmerz- und Palliativmedizin in Deutschland, der an den Verhandlungen beteiligt war.

Mit der Vereinbarung würden die Anforderungen an die aktuellen Vorgaben der Zusatzweiterbildung "Spezielle Schmerztherapie" angepasst, heißt es weiter in der Mitteilung. Auch die Praxisbedingungen würden nun flexibler gestaltet, etwa für größere Einrichtungen. Außerdem wurden die Modalitäten zur Dokumentationsprüfung verändert.

\section{"Endmenstruelle Migräne" \\ Ein neuer Kopfschmerztyp bei Frauen?}

— Wenn Frauen regelmäßig gegen Ende ihrer Periode über Kopfschmerzen klagen, ist das möglicherweise durch den Blutverlust bedingt. Neurologen aus North Carolina postulieren einen neuen Kopfschmerztyp: die „endmenstruelle“ Migräne [Calhoun AH et al. Headache 2016; online 5. Oktober]. Der Zusammenhang war den Forschern um Dr. Anne H. Calhoun vom Carolina Headache Institute in Durham schon vor einigen Jahren aufgefallen: So gab es unter den Frauen in ihrer Migräne-Ambulanz immer wieder Patientinnen, die die Kopfschmerzschübe nicht klassischerweise zu Beginn ihrer Periode sondern erst gegen Ende bekamen.

In der auf Menstruationskopfschmerzen spezialisierten Klinik fanden sich in einem Zeitraum von sechs Wochen 85 Frauen mit Migränediagnose, die einen regelmäßigen Zyklus hatten und damit für die Auswertung geeignet waren. 30 von ihnen $(35,3 \%)$ hatten angegeben, dass jeweils in den letzten Tagen ihrer Monatsblutung Kopfschmerzen einsetzten und dann im Schnitt 2,6 Tage anhielten. Für die Forscher ist das ein klarer Hinweis auf die Existenz der von ihnen postulierten „endmenstruellen“ Migräne (EMM). In der Migräne-Klinik werden bei allen Patientinnen routinemäßig die Ferritinwerte bestimmt. Diese lagen bei 28 der 30 EMM-Kandidatinnen unterhalb des allgemein akzeptierten Grenzwertes von $50 \mathrm{ng} / \mathrm{ml}$. Bei der Hälfte waren es sogar

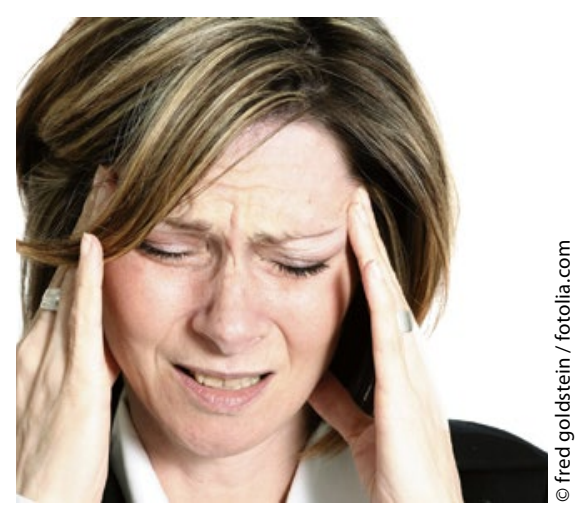

weniger als $18 \mathrm{ng} / \mathrm{ml}$. Ferritin dient im Organismus nicht nur als Speicherstoff für Eisen, sondern kontrolliert auch dessen Freisetzung. Mangelt es an dem Protein, können sich die Eisenspeicher schnell entleeren.

Wie Calhoun et al. zeigen konnten, stand die „EMM", anders als die "normale“ Menstruationsmigräne, nicht im zeitlichen Zusammenhang mit hormonellen Schwankungen, sondern ereignete sich in einer Phase sehr stabiler Östrogenkonzentrationen. Die Forscher postulieren, dass es bei Frauen mit ohnehin niedrigen FerritinSpiegeln durch den monatlichen Blutverlust zu einem ausgeprägten Missverhältnis zwischen dem Sauerstoffbedarf und der Menge an zirkulierenden roten Blutkörperchen komme.

(eo)

\section{Kopfschmerz bei Schülern Unterricht gegen Schmerz}

Viele Schüler haben wie selbstverständlich Kopfschmerztabletten im Ranzen. Das geht aus einer Umfrage unter 1.102 Siebtklässlern hervor. Demnach leiden knapp 74\% der Jugendlichen an Migräne oder Spannungskopfschmerzen. Davon nehmen drei Viertel regelmäßig Schmerzmedikamente ohne ärztliche Verordnung. Weniger als ein Drittel der Betroffenen weiß um die Diagnose. Sie nehmen ein, was da ist: ASS, Paracetamol oder lbuprofen. „Die meisten haben kein Konzept und keinen Namen für ihre Kopfschmerzen", sagte Professor Hartmut Göbel von der Schmerzklinik Kiel.

Gemeinsam mit der Lehrerin Karin Frisch vom gemeinnützigen Zentrum für Forschung und Diagnostik bei Implantaten, Entzündungen und Schmerzen (Zies $\mathrm{gGmbH}$ ) in Frankfurt hat Göbel die "Aktion Mütze - Kindheit ohne Kopfzerbrechen“ ins Leben gerufen. Diese stellt Schulen Unterrichtsmaterial zur Kopfschmerzprävention zur Verfügung. 21 gesetzliche Krankenkassen unterstützen das Projekt bislang mit rund 2,5 Millionen Euro.

„Durch die Unterrichtseinheit lernen Schüler, Eltern und Lehrer wie sie durch Veränderungen im Alltag Kopfschmerzen vorbeugen können", erklärte Frisch. Dazu gehöre regelmäßiger Schlaf, Bewegung, kohlenhydratreiche Mahlzeiten und ein bewusster Umgang mit Medien. Anno Fricke 